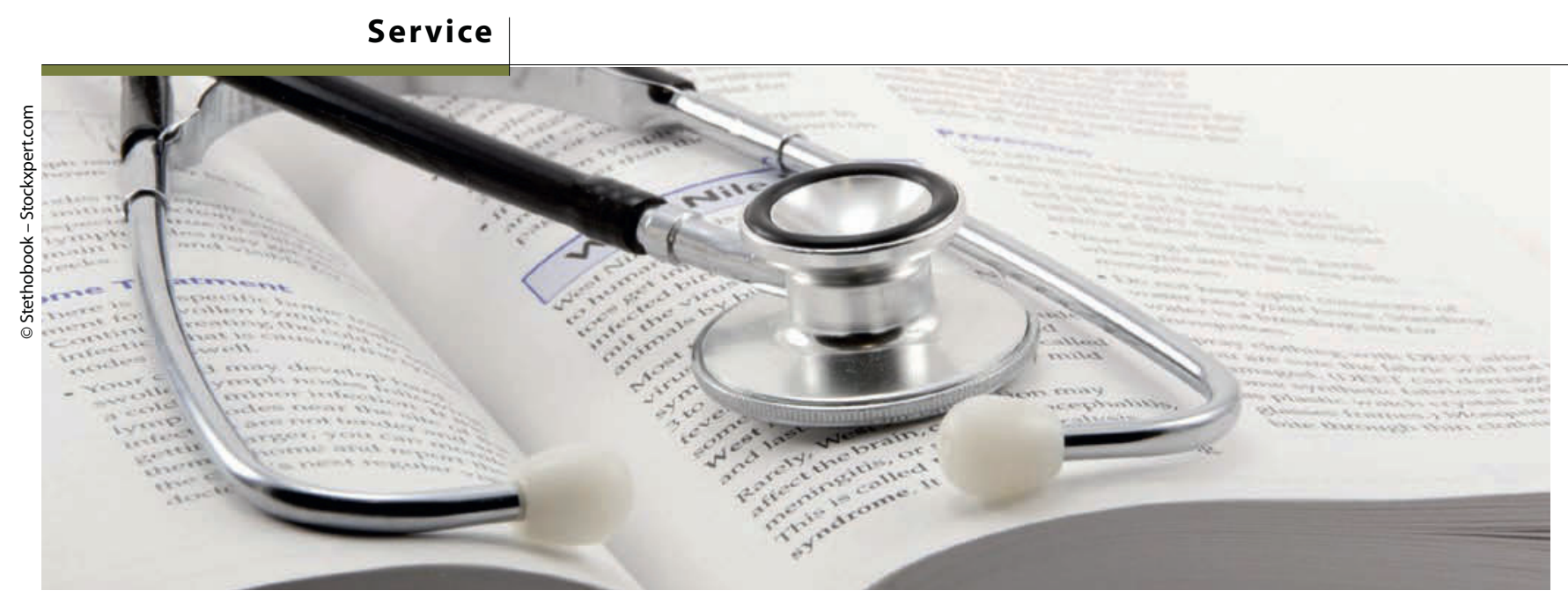

\title{
Buchtipps
}

\section{Neues auf dem Markt}

\author{
Nach wie vor ist das Buch aus \\ der Fort- und Weiterbildung \\ nicht wegzudenken. Aber immer \\ häufiger gibt es zu crossmediale \\ Komponenten, wie beispielswei- \\ se zusätzliche Downloads.
}

\section{Chronische Schmerzen - besser verstehen}

Das Buch dient der Therapie-Unterstützung. Betroffene und Angehörige werden dort abholt, wo sie stehen. Ohne falsche Hoffnungen zu wecken, können sie sich mit den neuesten medizinische Erkenntnissen vertraut machen. Die verschiedenen Schmerzerkran-

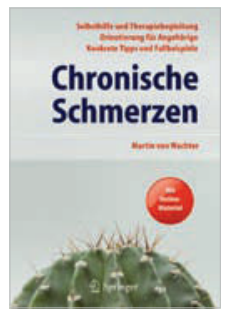

\section{Wachter}

Chronische Schmerzen Selbsthilfe und Therapiebegleitung - Orientierung für Angehörige - Konkrete Tipps und Fallbeispiele. Mit Online-Material 1. Auflage,

Springer Verlag 2012, ISBN 978-3-642-19612-6 $19,95 €$

kungen und ihre Behandlungsmöglichkeiten werden differenziert dargestellt, u. a. anhand von Fallbeispielen. Darüber hinaus gibt es Kontaktadressen, konkrete Tipps sowie eine Vielzahl von Online-Materialien: kostenloser Download von Arbeitsblättern und AudioEntspannungsübungen im Web. Erscheinen soll das Buch Ende September 2011.

\section{Ambulante Palliativversorgung}

"Wir möchten uns an all diejenigen wenden, die sich täglich in der Patientenversorgung an der Basis ambulant und stationär enga-

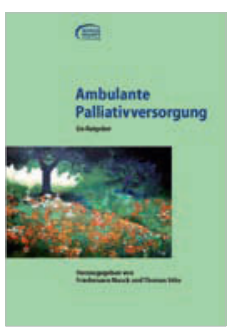

F. Nauck, Th. Sitte (Hrsg.) Ambulante Palliativversorgung - Ein Ratgeber Nachdruck der 1. Auflage, Deutscher Palliativ Verlag 2011, ISBN: 978-3-9814144-1-7 $4,99 €$

gieren. Auch an interessierte Entscheider in der Gesundheitsversorgung, Politik und ..." schreiben die Herausgeber. Der Ratgeber Ambulante Palliativversorgung beschäftigt sich zum einen mit dem aktuellen Stand der Palliativversorgung, der ambulanten Palliativversorgung und ihren speziellen Aufgaben sowie den grundlegenden Behandlungsansätzen in der Palliativmedizin. Darüber hinaus werden nochmals die 13 Achener Thesen aufgeführt, die Anfang 2010 im Rahmen einer Arbeitstagung erarbeitet wurden und zum Ziel haben, die Implementierung der Spezialisierten Ambulanten Palliativversorgung (SAPV) voranzutreiben.

\section{Schmerzpsychotherapie}

Die Schmerzpsychotherapie ist mittlerweile von allen führenden Fachgesellschaften anerkannt und fester Bestandteil eines modernen multimodalen Behandlungskonzeptes bei Schmerzpatienten. Das vorliegende Werk gilt als Standardwerk für die Fort- und
Weiterbildung Schmerzpsychotherapie und umfasst das Pflichtwissen zur Erlangung des Zertifikats „Psychologische Schmerztherapie“ der DGSS und DGPSF. Es zeigt praxisnah und verständlich die Vielfalt des Fachgebietes der Schmerzpsychotherapie, darunter auch spe-

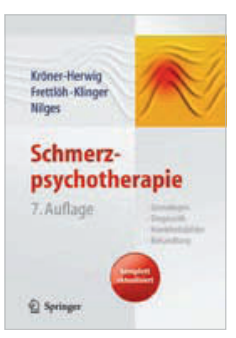

B. Kröner-Herwig, J. Frettlöh, R. Klinger, P. Nilges, (Hrsg.)

Schmerzpsychotherapie Grundlagen - Diagnostik - Krankheitsbilder Behandlung

7. vollst. überarb. Auflage Springer Verlag 2011, ISBN 978-3-642-12782-3 $64,95 €$

zielle Themen wie "Schmerz und Geschlecht", „Schmerz bei Migranten". Ein patienten- und praxisorientiertes Nachschlagewerk für alle klinisch tätigen Psychotherapeuten und Ärzte, die ihre Patienten fächerübergreifend behandeln.

\section{Schmerzen verlernen}

Betroffene mit andauernden Schmerzen, suchen häufig Alternativen und Ergänzungen zu Schmerzmitteln und medizinischer Therapie.

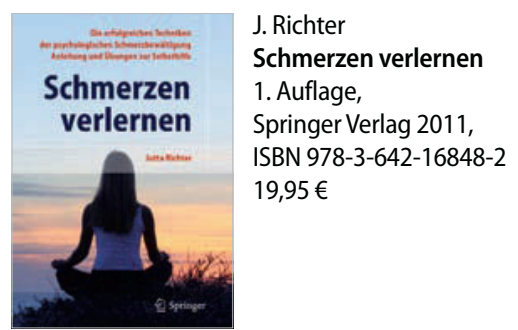

Dabei können Techniken der psychologischen Schmerzbewältigung eine hervorragende Hilfe sein. Das vorliegende Werk zeigt Ihren Patienten über 30 erfolgreiche Übungen/Techniken aus der Praxis, die sie gegebenenfalls auch alleine lernen und anwenden können. 